\title{
Coordinated Electron and X-ray Microscopy of Cometary Organic Matter Collected by the NASA Stardust Mission.
}

\author{
Bradley T. De Gregorio ${ }^{1}$, Rhonda M. Stroud ${ }^{2}$, Larry R. Nittler ${ }^{3}$, George Cody ${ }^{4}$, and A. L. David \\ Kilcoyne ${ }^{5}$ \\ 1. Nova Research Inc., Alexandria, VA USA. \\ 2. Materials Science and Technology Division, Naval Research Laboratory, Washington, DC USA. \\ 3. Department of Terrestrial Magnetism, Carnegie Institution of Washington, Washington, DC USA. \\ 4. Geophysical Laboratory, Carnegie Institution of Washington, Washington, DC USA. \\ 5. Advanced Light Source, Lawrence Berkeley National Laboratory, Berkeley, CA USA.
}

Comets contain remnant material left over from the formation of our Solar System about 4.6 billion years ago. Preserved cometary organic matter thus informs us about the initial organic content of the early solar nebula, from which eventually all life in the Solar System formed. Here we describe nano-scale coordinated electron and X-ray microscopy, and secondary ion mass spectroscopy for the study of the morphology, crystallinity, organic functional chemistry, and isotopic composition of carbonaceous matter in cometary dust particles collected by the NASA Stardust mission to Comet 81P/Wild 2.

Stardust particles were picked out of silica aerogel, embedded in sulfur droplets, and ultramicrotomed. Alternating microtome sections were also placed on other grids/substrates for characterization of inorganic components or to enable more robust isotopic measurements of the cometary particles. Samples were first analyzed using STXM at various synchrotron beamlines (X1A1 at the National Synchrotron Light Source, 5.3.2.2 at the Advanced Light Source, and 10ID-1 at the Canadian Light Source). X-ray absorption images with a spatial resolution of $30 \mathrm{~nm}$ were acquired at sequential energy steps to generate a hyperspectral image "stack", from which X-ray absorption near-edge structure (XANES) spectra are extracted. By targeting the $\mathrm{C}, \mathrm{N}$, and $\mathrm{O}$ absorption edges, the overall organic functional group chemistry of each sample was determined, in addition to an estimate of the N/C and $\mathrm{O} / \mathrm{C}$ content. The same grids were observed with the field emission JEOL 2200FS TEM at the Naval Research Laboratory. By correlating STXM and TEM images, functional chemistry indicated by XANES was matched to sample morphology and context to identify samples that have undergone alteration during capture or are likely contaminants. Finally, the H, C, and N isotopic composition of the same samples was measured with a Cameca NanoSIMS 50L ion microprobe at the Carnegie Institution of Washington to identify cometary organic matter with a probable extra-solar heritage.

A major finding of the preliminary Stardust examination is that Comet Wild 2 contains abundant aliphatic and N-rich organic matter [1,2]. All samples that were observed by STXM to contain predominantly aliphatic organic matter were shown by TEM to be intimately mixed with the silica aerogel or epoxy [3]. These samples are likely authentic cometary organic matter, but reliable information on their chemistry cannot be obtained. Two N-rich samples contained metal oxide nanoparticles, and are therefore likely contaminants [3]. Two additional types of functional chemistry were observed in the Stardust samples. The first consists of polyaromatic macromolecular material [3], similar in structure to terrestrial kerogen and other macromolecular organics in meteorites and interplanetary dust. In the cometary samples, however, this organic matter contains less aromatic carbon and more carbonyl functionality than typical meteoritic organic matter (Figure 1). The second type 
consists of highly aromatic, but not quite graphitic, organic matter (Figure 2) [3]. In two cases, the cometary organics took the form of organic nanoglobules [4], which are frequently observed in primitive meteorites and interplanetary dust [5]. In a third case, the highly aromatic organic matter formed core-shell structures around Fe-metal nanoparticles. NanoSIMS isotopic measurements of the samples confirmed the cometary, and possibly extra-solar, origin of some of the macromolecular and highly-aromatic organic particles $[3,4,6]$.

\section{References:}

[1] S Sandford et al, Science 314 (2006), p. 1720-1724.

[2] G Cody et al, Meteoritics \& Planetary Science 43 (2008), p. 353-365.

[3] B De Gregorio et al, Meteoritics \& Planetary Science 46 (2011), p. 1376-1396.

[4] B De Gregorio et al, Geochimica et Cosmochimica Acta 74 (2010), p. 4454-4470.

[5] B De Gregorio et al, Meteoritics \& Planetary Science 48 (2013), p. 904-928.

[6] This work was funded in part by the NASA LARS Program. The ALS and NSLS are supported by the US Department of Energy (Contract No. DE-AC02-05CH11231), and the CLS is supported by CFI, NSERCC, NRCC, CIHR, the Government of Saskatchewan, and the University of Saskatchewan. Dr. K. Nakamura-Messenger is thanked for performing some of the sample preparation in the Stardust Curation Laboratory at NASA Johnson Space Center.

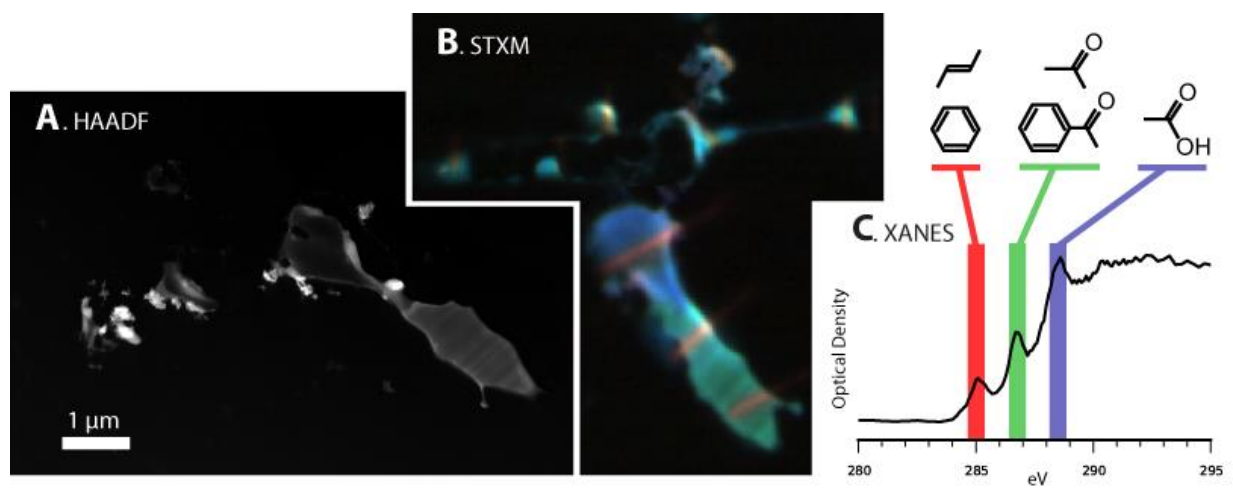

Figure 1. (A) High-angle annular dark field STEM image of an organic cometary particle. Bright areas are silica aerogel. (B) False color STXM image of an adjacent microtome slice. Red $=285 \mathrm{eV}$ intensity from aromatic $\mathrm{C}=\mathrm{C}$; Green $=286.7 \mathrm{eV}$ intensity from ketone functional groups; Blue $=288.6 \mathrm{eV}$ intensity from carboxyl functional groups. (C) Average XANES spectrum of the large organic fragment.
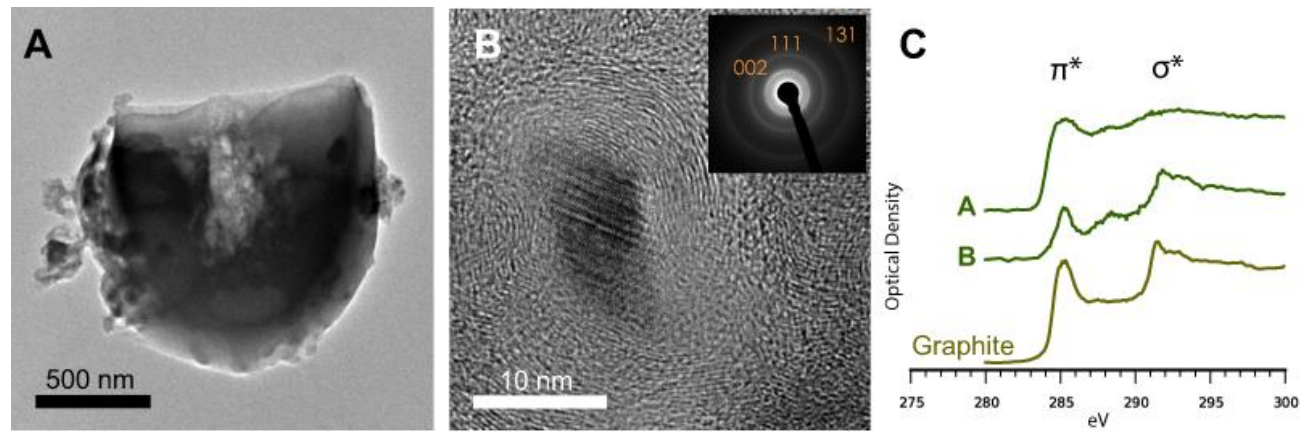

Figure 2. TEM images of highly aromatic cometary organic matter. (A) Spherical nanoglobule. (C) Poorly graphitized layers surrounding a core Fe-metal nanoparticle. Inset shows electron diffraction rings characteristic of graphite. (C) XANES of the two aromatic samples, compared to that of graphite. 